Wenn aber $\sin \beta v_{1}=0.113$ sein soll, so findet man $\beta v_{1}=6^{\circ} .5$. Der Winkel $\beta v_{1}$ wächst von $0^{\circ}$ bis $360^{\circ}$ in dem Zeitraum von $\mathbf{1 3 2 0 0 0}$ Jupitersumläufen, Derselbe erreicht daher den Werth 6:5 in dem Zeitraum von 2400 Jupitersumläufen. Ich habe in diesem Aufsatz die Störungen

Karlsruhe im April $₫ 884$. der ersten Ordnung entwickelt, welche die Uranusbahn durch den Jupiter erleidet. Bleibt der Bogen $\beta v_{1}$ zwischen den Grenzen \pm 6.5 , so geben diese Entwickelungen die Genauigkeit einer Bogensekunde und gelten für den Zeitraum ron beiläufig 4800 Jupitersumläufen.

\title{
Osservazioni della Cometa 1884 II (Barnard)
}

fatte nel R. Osservatorio di Brera a Milano.

\begin{tabular}{|c|c|c|c|c|c|c|c|c|c|}
\hline I 884 & I. M. Milano & $\Delta c$ & $\Delta d$ & Confr. & $\alpha$ app. & $\log p \cdot 1$ & $\delta$ app. & $\log p \cdot A$ & $*$ \\
\hline Sett. 20 & $7^{\mathrm{h}} 4 \mathrm{r}^{\mathrm{m}} 5^{6^{\mathrm{s}}}$ & $+2^{111} 5^{5} \cdot 3 x$ & $-3^{\prime} 23^{\prime \prime} 3$ & 8 & $19^{\mathrm{h}} 39^{\mathrm{m}} 20^{\mathrm{s}} 2 \mathrm{I}$ & $7.8 \mathrm{I} 6$ & $-27^{\circ} 58^{\prime} \quad 5^{\prime \prime} \cdot 9$ & 0.927 & I \\
\hline
\end{tabular}

Stelle di confronto.

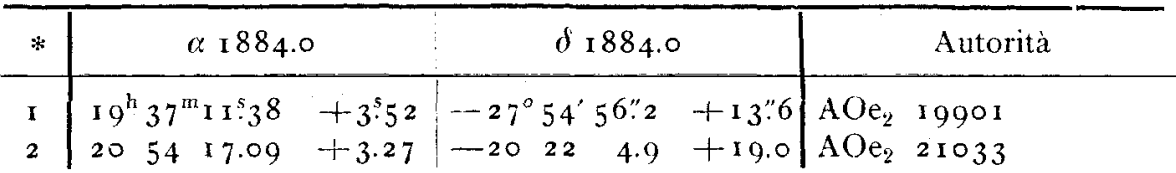

L'osservazione dell' I I Ottobre era alquanto penosa. Il 20 Ottobre ho veduto la Cometa, ma non era più osservabile col nostro strumento di 8 pollici.

Osservatorio Reale di Brera a Milano I 884 Novembre 15.

G. V. Schiaparelli.

\section{Beobachtungen des Cometen $1884 \mathrm{~W}^{\mathrm{T}}$ olf} auf der Sternwarte in Gohlis.

\begin{tabular}{|c|c|c|c|c|c|c|c|c|c|}
\hline 1884 & M. Z. Gohlis & $\Delta a$ & $\Delta \delta$ & Vergl. & $c$ app. & $\log p . \Delta$ & $\delta$ app. & $\log p .4$ & $*$ \\
\hline Oct. I 0 & $9^{\mathrm{h}} 39^{\mathrm{m}} 3^{8^{\mathrm{s}}}$ & $-0^{\mathrm{m}} 19^{\mathrm{s}} \cdot 72$ & - $15^{\prime} 24^{\prime \prime} 6$ & 5 & $2 \mathrm{I}^{\mathrm{h}} 32^{\mathrm{m}} 3^{8 .} .74$ & 9.146 & $+13^{\circ} 54^{\prime} 48^{\prime \prime} .9$ & 0.747 & I \\
\hline 20 & 85222 & -030.12 & - I 24.7 & 5 & $2147 \quad 45.67$ & 9.010 & +8856.0 & 0.734 & 2 \\
\hline 23 & $\begin{array}{lll}9 & 35 & 19\end{array}$ & to 43.34 & - I 344.6 & I 4 & $2153 \quad 5.95$ & 9.243 & +64633.4 & 0.797 & 3 \\
\hline 23 & I $05^{6} 4^{6}$ & -451.75 & +758.6 & 6 & 2 I 53 I 2.00 & 9.433 & +6453.8 & 0.807 & 4 \\
\hline Nov. 7 & $\begin{array}{lll}741 & 33\end{array}$ & $+4 \times 2.59$ & +526.1 & Io & 2337.00 & 8.619 & $+\circ 5313.8$ & 0.834 & 5 \\
\hline 8 & $730 \quad 1$ & 一o 57.84 & $+\quad 145.1$ & 20 & 2551.02 & 8.453 & $+\circ 3^{2} 3^{8.9}$ & 0.837 & 6 \\
\hline
\end{tabular}

Mittlere Oerter der Vergleichsterne für I884.0.

\begin{tabular}{|c|c|c|c|c|c|c|}
\hline$*$ & \multicolumn{2}{|c|}{$\alpha \quad$ I 884.0} & \multicolumn{3}{|c|}{$\delta$ I 884.0} & Autorität \\
\hline I & $2 \mathrm{I}^{\mathrm{h}} 32^{\mathrm{m}} 55^{\mathrm{s}} \cdot 2 \mathrm{I}$ & +3.25 & $+13^{\circ}$ & 43.8 & +29.8 & Glasg. $\quad 5534$ \\
\hline 2 & $2148 \quad 12.58$ & +3.21 & +8 & 52.6 & +28.8 & $\therefore \quad 5619$ \\
\hline 3 & $5^{2}$ i 9.40 & +3.21 & +65 & $5 \mathrm{I} .6$ & +28.4 & Schjell. 8949 \\
\hline 4 & $\begin{array}{lll}21 & 5^{8} & 0.49\end{array}$ & +3.26 & +63 & 36.9 & +28.4 & \# 8998 \\
\hline 5 & 221920.98 & +3.13 & +04 & 21.6 & +26.1 & ${ }^{1 / 2}\left(\right.$ Schjell.9166 + New 7 yr. $\left.25^{6} 3\right)$ \\
\hline 6 & $22 \quad 29 \quad 45.68$ & +3.18 & $+\circ 3$ & 27.9 & +25.9 & Schjell. 92 I 8 \\
\hline
\end{tabular}

Bemerkungen.

Oct. 10. Beobachtung unsicher. Antritte nur am Rande des Gesichtsfeldes $\left(r=829^{\prime \prime 4} 8\right)$.

Oct. 20. $\Delta \delta$ unsicher. Comet erscheint als runde Nebelmasse mit centraler Verdichtung. Helligkeit 9. Gr.

Nov. 8. Beobachtung schwierig. Dunstige Luft. Comet als Nebelmasse ohne Andeutung von Kern. Helligkeit ro. Gr. 